\title{
Preface to Special Edition of EM\&P, Reporting on the INAF/Arcetri-ESO-Workshop on "Future Ground-based Solar System Research: Synergies with Space Probes and Space Telescope" held at Portoferraio, Elba, Italy, 8-12 September 2008
}

\author{
Hans Ulrich Käufl · Gian Paolo Tozzi
}

Received: 14 July 2009/Accepted: 14 July 2009/Published online: 25 July 2009

(C) Springer Science+Business Media B.V. 2009

In 2006 there was a dedicated workshop, co-sponsored by ESO together with the Vrije Universiteit Brussel, to derive synergies from the spectacular world wide coordinated observing campaign in the context of NASAs Deep Impact space mission to comet 9P/ Tempel 1 (Käufl and Sterken 2006, 2009; Meech et al. 2005). This comet had been at the focus of an unprecedented worldwide long-term multi-wavelength observation campaign. Many participants at this workshop, held in Brussels, beyond their direct involvement in the Deep Impact experiment, frequently expressed how useful it was to hold interdisciplinary workshops, which bring together Solar System researchers, involved in spacecraft experiments, ground- and space-based remote observers and theoreticians. The idea of a similar meeting was born. Part of the program of the Brussels' workshop was a joint excursion to the battle ground of Waterloo, close to the conference venue. Standing on the monument there, some participants reasoned, how incredible it was, that this battle took place only 100 days after Napoleon's escape from the island of Elba, and how difficult it often is these days to get anything going within a few months. Somehow this sparked the idea of the "Route Napoleon Reverse" that is to say, the next such workshop should happen on the island of Elba. Needless to say, it took us more than 100 days to organize it!

In the coming decades fundamentally new observing platforms and space probes will become available for Solar System research. The Elba 2008 workshop provided a forum to discuss the use of these future facilities, especially to optimize the scientific returns and to establish synergies. Particularly interesting was to identify, or at least start a process to identify, potential paradigm-shifting observations that will become possible with the next generation of large ground-based telescopes and their advanced instrumentation (Fig. 1).

Among the various goals of the workshop fostering of collaborations between ground and space projects, such as between ESO and ESA ground space was the primary goal. In general we sought to create synergies between research at different wavebands of Solar System objects. For the ground-based projects the aims were to define the Extremely Large

H. U. Käufl $(\bowtie)$

ESO, Karl Schwarzschild Strasse 2, 85748 Garching bei München, Germany

e-mail: hukaufl@eso.org

G. P. Tozzi

INAF-Osservatorio Astrofisico di Arcetri, Largo E. Fermi 5, 50125 Florence, Italy 


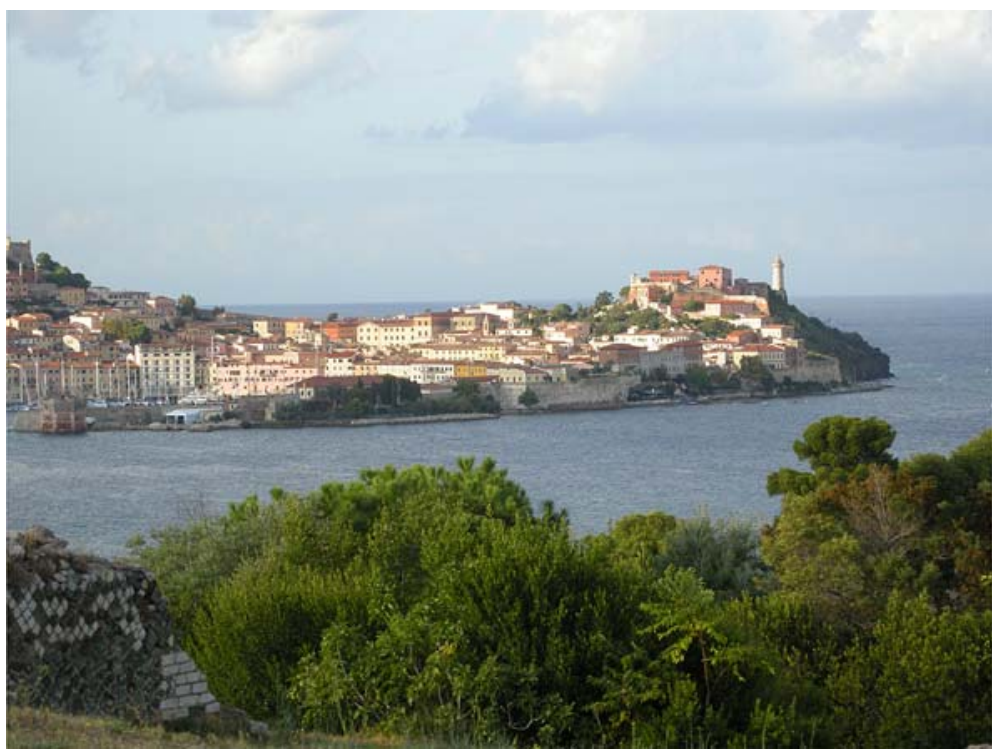

Fig. 1 The workshop took place in the historic center of the city of Portoferraio, dominated by the Renaissance fortifications erected in the 16th century under the reign of Cosimo I de Medici. The venue, nearly in the center of the photograph, flanked to the left by a building of reddish ochre colour, to the right by a small church tower, was then originally commissioned as a barracks. It became later a monastery but serves now as a cultural and Congress centre (named after Cesare De Laugier), as well as a picture gallery, (Pinacoteca Foresiana) and the city library (Biblioteca Comunale)

Telescope (ELT), and in particular the European ELT (E-ELT), science cases for the Solar system science and to refine the science case for the Atacama Large Millimetre/Submillimetre Array (ALMA).

The topics that were specifically addressed during the meeting fall under the two headings of ground-based support for existing or planned space missions and new facilities for remote observations. There are missions to comets and asteroids (e.g. Rosetta, Dawn, Deep Impact and Stardust Wrap-up), to the outer Solar System planets and moons (e.g. Cassini/Huygens and New Horizons), to terrestrial planets and the Moon (e.g. Mars and Venus Express, Messenger and BepiColombo). In the ESA Cosmic Vision 2015-20 Programme there are also expected to be a number of planetary missions. In the area of new facilities for remote observations the planned ELTs (E-ELT, Thirty Meter telescope (TMT) and Giant Magellan telescope (GMT) were considered as well as ALMA.

Following a first series of reviews and status reports on the major observing facilities, topical sessions were held on main belt asteroids, giant planets including their moons and magnetospheres, Trans-Neptunian Objects (TNOs) (including Pluto), comets and the formation of the Solar System. A bridge was made from the study of our Solar System to the relatively new field of extrasolar planetary systems. We start to consider our Solar System as one of many possible planetary systems, or alternatively, our Solar System to be in range of extrapolation of current theories of star and planetary system formation. Our Solar System is also the yardstick to define the characteristic observables when direct observations of extrasolar planets become feasible, with future facilities such as the E-ELT. 
The detailed scientific program of the meeting is available at www.arcetri.astro. it/elba2008/. The scientific scope ranged from the detection of the tenuous sodium atmospheres of Mercury and our Moon to bio-signatures of extra-solar planets.

In the conference summary, provided by Hermann Boehnhard, the following prime conclusions were reached and agreed:

- even the most advanced and sophisticated space missions that provide for in situ data need the complement of remote sensing data in order to place the observations in their wider scientific context;

- the Solar System inventory is far from complete and there is a strong need for more surveys. For the faintest objects a serendipitous occultation mode (e.g. involving telescope acquisition cameras) offers great promise;

- as concerns the inventory of asteroid and cometary nuclei, systematic statistical studies of shapes, sizes, albedos and rotation depend critically on Earth-based telescopes as well as the James Webb Space telescope (JWST). The same conclusion holds for the study of their surface chemistry;

- paradigm-changing observations can, for example, be expected in the field of planetary atmospheres. Currently the long-term stability of planetary atmospheres against erosion by solar UV radiation and particle flux is not understood; high resolution spectral and spatial observations may provide for fundamentally improved insights into the relevant processes;

- in order to achieve a synthesis between the observations and theory of extra-solar proto-planetary discs and our Solar System, more mineralogical data (e.g. mid-infrared low resolution spectroscopy) for primitive bodies in our Solar System are mandatory;

- European Astronomers will be in the front seat of these research programs thanks to participation in ALMA and the instrumentation suite under study for the E-ELT (D'Odorico et al. 2008).

In a splinter session, some 20 participants also convened to discuss how to promote Solar System observations in the Science Case of the E-ELT with a special Solar System section. Meanwhile ESO had launched an inquiry with its users to submit contributions to the E-ELT Design Reference Science Plan (DRSP). While a detailed analysis of the proposed science cases is still pending-deadline for submission was June 1st 2009-we know, that at least 12 colleagues have come up with a proposal for Solar System observations for the Science Plan of the E-ELT.

Last but not least we wish to recall the question raised by Thomas Mueller: "Is the Asteroid (2567) Elba smaller or bigger than (the island of) Elba?". Meanwhile the answer has been put forward by Thomas: "The island Elba has about $224 \mathrm{~km}^{2}$ corresponding to an effective diameter of about $16.9 \mathrm{~km}$. (2567) Elba has an H-magnitude of $11.8 \mathrm{mag}$, corresponding to a possible diameter range between 8 and about $35 \mathrm{~km}$, depending on its albedo. This asteroid was not seen by IRAS, ISO, MSX, Spitzer. It has no adaptive optics observations and no N-/Q-band observations. Now, we have found it in five independent scans of the Akari-satellite at 18 micron. A simple model calculation resulted in an effective diameter of about $30 \mathrm{~km}$ and a geometric albedo of 0.04. (2567) Elba is therefore much bigger than Elba, but at the same time it seems to be a very dark and boring place compared to the real island...." 


\section{References}

S. D'Odorico et al., SPIE 7014, 70141 (2008)

H.U. Käufl, C. Sterken, The Messenger 126, 48 (2006)

H.U. Käufl, C. Sterken, in Deep Impact as a World Observatory Event: Synergies in Space, Time, and Wavelength: Proceedings of the ESO/VUB Conference, Brussels, Belgium, 7-10 August 2006. ESO Astrophysics Symposia, Volume. ISBN 978-3-540-76958-3. Springer, Berlin, Heidelberg 2009

K. Meech et al., Science 310, 265-269 (2005) 